\title{
Emphysematous cystitis: The role of CT imaging and appropriate treatment
}

\author{
Alper Eken, $M D$, FEBU; ${ }^{*}$ Ergun Alma, $M D^{+}$ \\ *Acabadem Adana Hastanesi Urology Department, Adana, Turkey; ${ }^{\dagger}$ Ceyhan State Hospital, Adana, Turkey
}

Cite as: Can Urol Assoc J 2013;7(11-12):e754-6. http://dx.doi.org/10.5489/cuai.472

Published online November 8, 2013.

\section{Abstract}

Emphysematous cystitis is a relatively rare disease characterized by the presence of gas in the bladder wall and/or lumen. The primary risk factor is diabetes mellitus. Emphysematous cystitis should be considered in cases of urinary tract infections in diabetic patients with unusual presentations. Imaging studies are necessary to detect emphysematous cystitis. Accurate diagnosis of the disease and appropriate treatment typically results in a favourable prognosis and can improve the outcome. We present a case of emphysematous cystitis diagnosed by a computed tomography scan in a diabetic woman with poor glycemic control.

\section{Introduction}

Emphysematous cystitis is a relatively rare and complicated urinary tract infection (UTI) primarily observed in diabetic middle-aged women. This disease is often characterized by non-specific clinical symptoms, with little or no diagnostic clues. It is defined by the presence of air within the bladder wall and/or the bladder lumen in imaging studies. ${ }^{1}$ Diabetes mellitus (DM) is the major risk factor of emphysematous cystitis. Other risk factors include neurogenic bladder, urinary tract outlet obstruction, chronic UTIs, indwelling urethral catheters, and immune-deficiency. ${ }^{2}$ Successful management of the disease and appropriate treatment with broad-spectrum antibiotics usually result in a favourable prognosis. ${ }^{1,2}$

\section{Case report}

A 65-year-old female was admitted to our department with a 2-day history of fever. She complained of nausea, vomiting and mild right flank pain for 3 hours and symptoms of urgency and dysuria for 7 days; she denied using antibiotics for these symptoms. She had a medical history of DM for 14 years and extracorporeal shock wave lithotripsy (ESWL) for a kidney stone 2 years ago. Despite using oral antidiabetics, she had poor diabetic control (fasting blood glucose was between 250 and $350 \mathrm{mg} / \mathrm{dL}$ ). She was conscious and interactive during the physical examination, which revealed an axillary temperature of $38.7^{\circ} \mathrm{C}$, arterial blood pressure of $130 / 70 \mathrm{mmHg}$, respiratory rate of 22 breaths per minute and oxygen saturation of $97 \%$. There was no guarding or rebound to abdominal palpation, but minimal suprapubic tenderness and right side costovertebral angle tenderness was noted.

Laboratory testing revealed the following abnormal results: serum white blood count 22,000/ $\mu \mathrm{L}$; erythrocyte sedimentation rate $60 \mathrm{~mm} / \mathrm{h}$; C-reactive protein $30.2 \mathrm{mg} / \mathrm{dL}$; blood urea nitrogen $95 \mathrm{mg} / \mathrm{dL}$; creatinine $2.4 \mathrm{mg} / \mathrm{dL}$; initial blood glucose level $380 \mathrm{mg} / \mathrm{dL}$ and $\mathrm{HbA} 1 \mathrm{C} \quad 9.2 \%$. The urine analysis revealed 60 to 80 red blood cells per field, significant leukocyturia and bacteriuria. Ketones, leukocyte esterase, nitrite and urine sugar were also positive (Table 1). Because of her kidney stone history and right flank pain, abdominopelvic non-contrast CT was performed; abdominal CT revealed a right kidney stone (Fig. 1), and pelvic images showed the presence of intraluminal gas, diffuse thickening of the bladder wall and gas in the bladder lumen (Fig. 2, Fig. 3). There were no findings of ureteral obstruction or pyelonephritis. The diagnosis of emphysematous cystitis was made based on the CT.

The patient started on intravenous tazobactam/piperacillin (TAZ/PIPC) after blood and urine cultures were obtained, and she was catheterized with an $18 \mathrm{Fr}$ Foley catheter. Glycemic control was established with insulin therapy. After 3 days, more than $1 \times 10^{6} \mathrm{CFU} / \mathrm{mL}$ Escherichia coli, sensitive to TAZ/PIPC, grew in the urine culture, but hemoculture was negative. The patient's clinical status improved; she became afebrile on day 5 and her catheter was removed. A repeat urine culture was sterile on the 18th day of the 


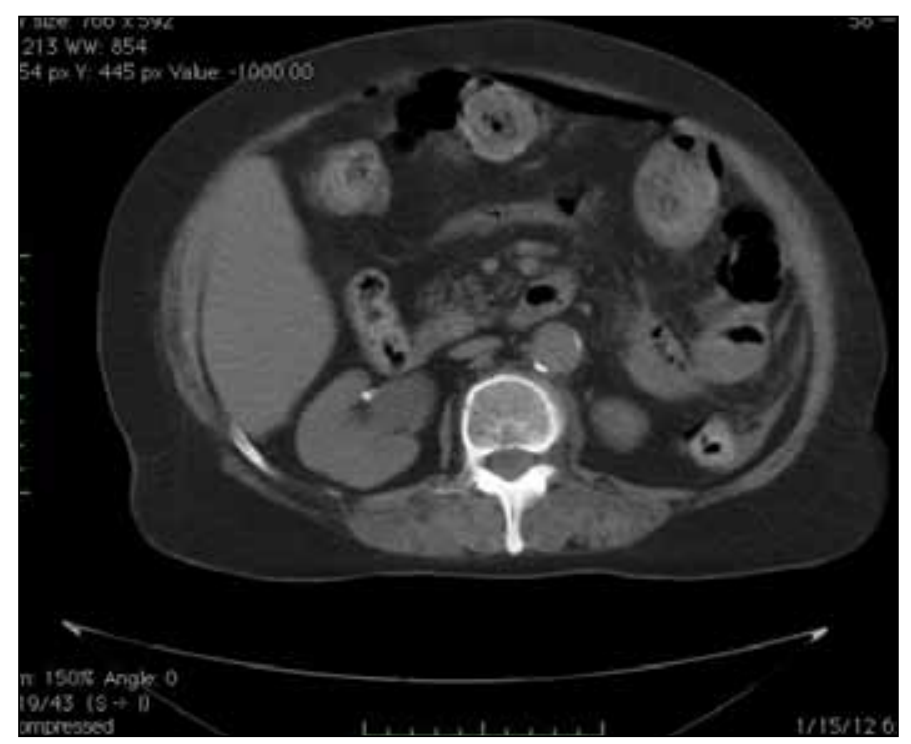

Fig. 1. Abdominal computed tomography demonstrating right kidney stone and mild hydronephrosis in the right kidney.

antibiotic treatment. Serum creatinine decreased from 2.4 to $1.8 \mathrm{mg} / \mathrm{dL}$; no additional decrease was observed. The TAZ/ PIPC was stopped on the 21 st day, a 2-week oral regimen of levofloxacin was started and she was discharged. At followup 3 weeks after discharge, the patient's urine analysis and culture were normal. Pelvic sonography revealed normal bladder wall thickening and no intraluminal gas.

\section{Discussion}

Emphysematous cystitis is rare, but potentially fatal if not treated properly. ${ }^{3}$ It presents similar to uncomplicated cystitis, which is characterized by dysuria, hematuria, abdominal pain, and urinary urgency and frequency with a pathogenic exception, but with the unique presence of pneumaturia. ${ }^{5}$ Varied presentations exist, such as incidental diagnosis in asymptomatic patients during abdominal imaging, subcutaneous emphysema and/or severe sepsis. ${ }^{1-4}$ Some patients do not recognize pneumaturia, while others are unwilling to acknowledge the condition. Diagnosis, which has gradually evolved to relaying on radiographic procedures rather than clinical ones, is critical.

The exact mechanism of pneumaturia is unclear and poorly understood. However, in diabetic patients, carbon may be produced through fermentation by the pathogens when glucose concentrations are high. Since the infection also affects non-diabetics, it is thought that tissue proteins and urinary lactulose can also serve as substrates. ${ }^{6}$ Impaired gas transport due to local inflammation or obstructive processes (which increase intracellular pressure while simultaneously decreasing circulation) may also increase the risk of pneumaturia. The release of bacterial endotoxin in complicated UTIs may contribute to the inflammatory pro-

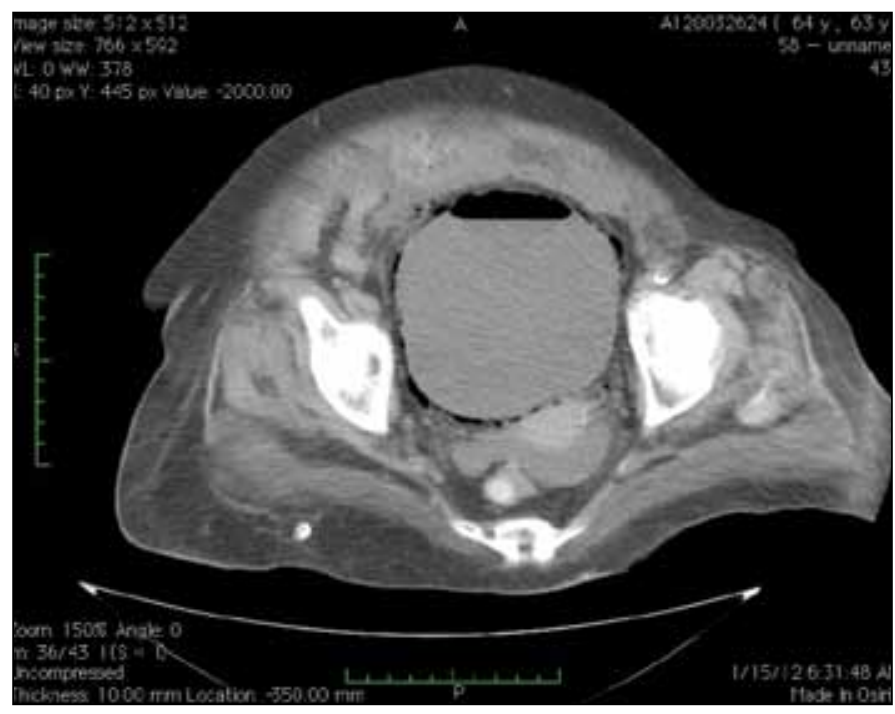

Fig. 2. Pelvic computed tomography demonstrating emphysematous changes in the bladder wall.

cess and may induce urinary tract paralysis and urinary stasis. ${ }^{1,2,5,7}$ Pneumaturia is highly suggestive, but rarely reported by patients. Vaginal fistula, colovesical fistula (particularly due to diverticular disease), Crohn's disease or carcinoma of the colon or bladder, instrumentation and after trauma are other causes of gas production, and differential diagnosis should be made with emphysematous cystitis. ${ }^{8}$

The prevalence and incidence of emphysematous cystitis are unknown. Patients who have obstructive uropathy, immunodeficiency, neurogenic bladder, and recurrent UTIs are at high risk..$^{1,3}$ Emphysematous cystitis may have an atypical presentation, and sometimes the degree of inflammation is not related to the symptoms. Because it is most common in patients with DM, clinicians should be alert to signs of these potentially fatal infections. Since radiographic imaging is necessary for the diagnosis of emphysematous cystitis, an abdominal plain film (at minimum) should be ordered if the disease is suspected in diabetic patients or in cases of UTIs with unusual presentations. These images may reveal a radiolucency outlining the bladder wall with or without luminal gas. Ultrasound scan may show abnormal and thickened bladder wall, but has a low sensitivity; it may be useful in patients who show clinical improvement at follow-up. ${ }^{9}$ However, CT is required for a definitive diagnosis and will allow differentiation from other causes. ${ }^{1,2,6}$

As observed with emphysematous pyelonephritis, about two-thirds of emphysematous cystitis infections are caused by E. coli; the remaining third are caused by Enterobacter species, Clostridium species, Klebsiella pneumonia, Staphylococcus aureus, or Proteus mirabi. ${ }^{1,3,10}$ Candida species may also be responsible. ${ }^{8} E$. Coli frequency is low in both emphsematous cystitis and pyelonephritis compared to common UTIs. ${ }^{1,3,10}$ Both aerobic and anaerobic cultures are required for identification of the etiology. Toyota and 


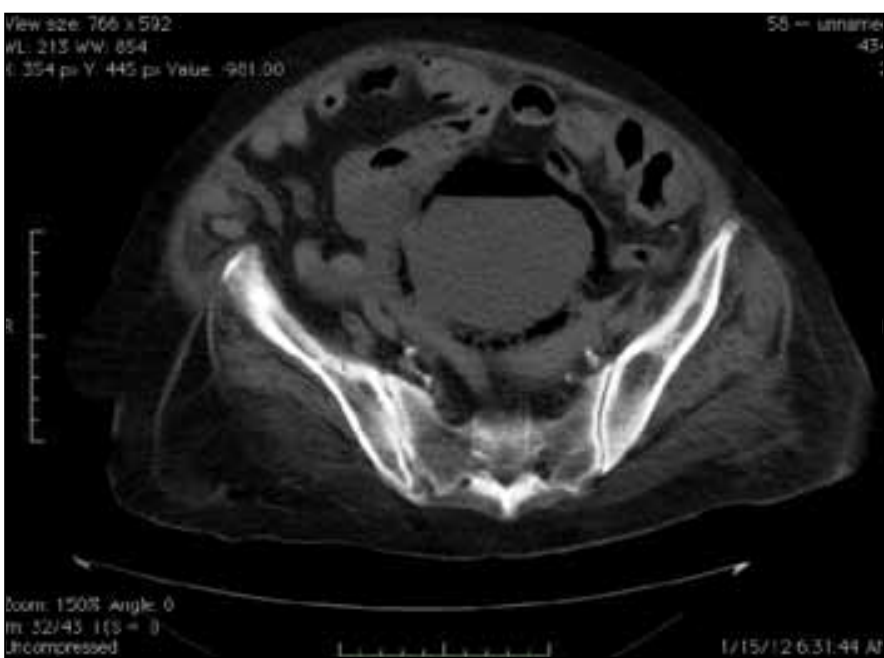

Fig. 3. Pelvic image showed presence of intraluminal gas and diffuse thickening of the bladder wall and gas in the bladder lumen.

colleagues reviewed the outcomes of 153 cases. The mean age of the patients was 62.7 , and most were women (63.4\%). DM was present in $66.7 \%$. The most common pathogens were E. coli and K. pneumonia, followed by Enterococci. The overall death rate was $6.6 \% .^{3}$

Endovenous antibiotics, such as flouroquinolones, penicillin with beta-lactamase inhibitors and third generation cefalosporines, are some of the appropriate treatments. Antifungal agents may be used in cases of a fungal infection. The agent in our case was E. coli, which was sensitive to piperacillin-tazobactam. Surgical interventions, such as debridement or partial cystectomy, may be required for patients who respond poorly to antibiotics or have necrotizing tissue. ${ }^{11,12}$ Described surgical indications include obstruction, bladder stone or anatomic abnormalities. ${ }^{5,8} \mathrm{~A}$ delay in diagnosis may cause bladder rupture, septicemia, peritonitis and death. Failure to recognize or diagnose this condition early in the course of the infection increases the associated mortality rate by up to $10 \% .^{1,3,8}$

\section{Conclusion}

Due to the high mortality rate of emphysematous pyelonephritis, prompt diagnosis is critical and can improve the outcome. Diagnosis should be followed by the appropri- ate combination of medication and surgical intervention, if needed. Radiological studies, especially CT, are important to achieve an early and accurate diagnosis. Although this disease is rare, it merits careful attention, especially in diabetic patients.

Acknowledgements: We are grateful to Associate Professor Tamer C. Inal, M.D., for editing the report.

Competing interests: Dr. Eken and Dr. Alma declare no competing financial or personal interests.

This paper has been peer-reviewed.

\section{References}

1. Thomas AA, Lane BR, Thomas AZ, et al. Emphysematous cystitis: a review of 135 cases. BJU Int 2007;100:17-20. http://dx.doi.org/10.1111/i.1464-410X.2007.06930.x

2. Mokabberi R, Ravakhah K. Emphysematous urinary tract infections: diagnosis, treatment and survival (Case review series). Am J Med Sci 2007;3331 1 1-6. http://dx.doi.org/10.1097/00000441-20070200000009

3. Toyota $\mathrm{N}$, Ogawa D, Ishii K, et al. Emphysematous cystitis in a patient with type 2 Diabetes mellitus. Acta Med Okayama 2011;65:129-33.

4. Sadek AR, Blake H, Mehta A. Emphysematouscystitis with clinical subcutaneous emphysema. Int J Emerg Med 2011;4:26.

5. Quint HJ, Drach GW, Rappaport WD, et al. Emphysematous cystitis: a review of the spectrum of disease. J Urol 1992;147:134-7.

6. Grupper M, Kravtsov A, Potasman I. Emphysematous cystitis: illustrative case report and review of the literature. Medicine 2007;86:47-53. http://dx.doi.org/10.1097/MD.0b013e3180307c3a

7. Dhingra KR. A case of complicated urinary tract infection: Klebsiella pneumoniae emphysematous cystitis presenting as abdominal pain in the emergency department. West J Emerg Med 2008;9:171-3.

8. Kumar A, Turney JH, Browniohn AM, et al. Unusual bacterial infections of the urinary tract in diabetic patients-rare but frequently lethal. Nephrol Dial Transplant 2001;16:1062-5. http://dx.doi.org/10.1093/ $\mathrm{ndt} / 16.5 .1062$

9. Middela S, Green E, Montague R. Emphysematous cystitis: radiological diagnosis of complicated urinary tract infection. BMJ Case Rep 2009;2009:bcr 05.2009.1832. Epub 2009 Dec 9.

10. Shigemura K, Yasufuku T, Yamashita $M$, et al. Bilateral emphysematous pyelonephritis cured by antibiotics alone: a case report and literature review. Jpn I Infect Dis 2009;62:206-8.

11. Perlmutter AE, Mastromichaelis M, Zaslau S. Emphysematous cystitis: a case report and literature review. W V Med J 2004;100:232-3.

12. Selley JK, Kane BG, Kane KE. Importance of obtaining a detailed medical history in diagnosing emphysematous cystitis. J Am Osteopath Asso 2010;110:91-4.

Correspondence: Dr. Alper Eken, Acibadem Adana Hastanesi Urology Department, Cumhuriyet Cad No 66, Postal Code: 01130 Adana, Turkey; fax:+903224554400; alpereken@yahoo.com 